\title{
Drugs and professional interactions: the modern day pharmacist
}

\section{Petty}

Heart 2003;89(Suppl II):ii31-ii32

Pharmacists are not yet fully integrated into the primary health care team and their skills could be better used to help patients with their long term medicines. The government is encouraging the setting up of medicines management services. Community pharmacists are well placed to help patients who have cardiovascular disease or who are at risk of this: they have an opportunity to identify at-risk patients, they can identify under treated patients at the point of dispensing, and they can provide education and advice on lifestyle and diet. They are also involved in smoking cessation services. Practice based pharmacists can improve patient care through medication review clinics.

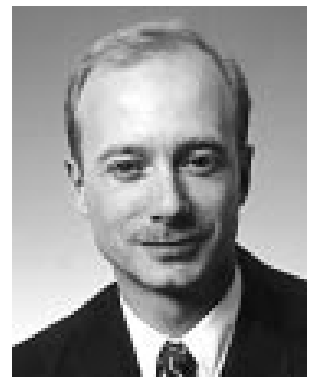

O ne of the major issues in the National Health Service (NHS) is said to be lack of human resources. Yet there are 23000 practising community pharmacists in Great Britain who are at present not being fully utilised in the health service. Pharmacists could be better used to help all patients who are taking long term medication. The pharmacy work environment is changing and, as well as practising as community pharmacists, pharmacists are working in primary care as public health pharmacists, as primary care trust pharmaceutical advisers, and, increasingly, as practice based pharmacists. At present, community pharmacists are funded to dispense medicines but negotiations are under way to revise the national contract to provide funding for professional services other than dispensing.

The profession is moving from purely product supply to "managing medicines". Pharmacy in the future-implementing the NHS Plan, ${ }^{1}$ the government's programme for pharmacy in the NHS which was published in 2000, highlights the need for pharmacists to help patients get the best from their medicines. It encourages medicines management services, which involve identifying and addressing unmet pharmaceutical need-for example, through medication review. This requires the pharmacist to spend time with patients, identifying under treated disease for example, and making sure that patients receive the correct treatments.

"Patient partnership" in medicine taking is also high on the government's and the profession's agenda. This means helping patients to reach decisions about treatments that they want to take, explaining the condition and treatment options, and discussing risks and benefits of taking and not taking medicines. As part of the concept of patient partnership, community pharmacists can be a source of education to patients about their treatments (table 1). They can explain duncan@

petty29.freeserve.co.uk the risks and benefits of treatment, side effects, drug-drug and drug-food interactions, and can discuss the monitoring requirements and what action the patient should take if symptoms occur.

\section{ACCESS TO SERVICES}

Integral to medicines management is better access to services. One aspect of this is the promotion of self care, with pharmacists helping individuals to cope with everyday health problems. Another access issue is the planned introduction of "instalment dispensing". This will be an important change for general practice in the UK. Under this scheme, the general practitioner (GP) writes a prescription, valid for six months, which the patient can then have dispensed in several instalments rather than having to go back to the surgery each time for a new prescription. When the patient collects their medicines, pharmacists will have the opportunity to confirm that the prescription still meets their needs and to supply any information the patient requires about their medicines.

Community pharmacists are well placed to help patients who have cardiovascular disease or who are at risk of this. They have an opportunity to identify at-risk patients, based on their knowledge of the families, what drug treatments are being taken, and information provided by patients. They can identify under treated patients at the point of dispensing and feed relevant information back to the surgery. Public health is another important aspect of the community pharmacist's work and involves provision of education and advice on lifestyle and diet. Pharmacists are also involved in smoking cessation services, and are able to support patients by providing structured advice and smoking cessation products. Some pharmacies participate in screening services-for example, measuring cholesterol and blood pressure. This can be useful provided that screening is undertaken in the context of global risk assessment and that services are discussed with local GPs to secure agreement on such matters as when patients should be referred for medical advice.

Table 1 Patient partnership in medicine taking

- Education

Risks and benefits of treatment Side effects

Drug-drug and drug-food interactions Requirements for monitoring and what to do if symptomatic

- Ongoing support Re-emphasis of above factors 


\section{Table 2 Compliance issues}

- Intentional non-compliance Informed decision not to take medicine III informed or un-informed decision not to take medicine

- Non-intentional non-compliance Cannot or forgets to order medicines Cannot open containers Gets confused with polypharmacy

\section{ENCOURAGING COMPLIANCE}

Another area in which pharmacists can help patients is in encouraging compliance. Issues can arise with both intentional non-compliance and non-intentional non-compliance (table 2). In intentional non-compliance, the patient has made a decision not to take a treatment. This may be an informed decision or it may be that the patient is misinformed or does not have the correct information. With education and advice, the pharmacist might be able to change the patient's behaviour. Non-intentional non-compliance is a major problem and arises when, for example, the patient forgets to take their medicine or becomes confused by their polypharmacy. Advice can be offered on making the medication regime simpler for patients. Some non-compliance relates to such basic issues as the patient not being able to open the medicine container.

To help improve compliance, it is essential that pharmacists, like doctors and nurses, provide ongoing support and use every opportunity to re-emphasise the importance of taking prescribed treatment.

\section{PRACTICE BASED PHARMACISTS}

A growing area of employment for pharmacists is work based in GP practices. One way in which practice based pharmacists can support patients is through undertaking audit to identify under treated or under monitored patients. Searches can be made by Read coding to identify specific groups of patients whose treatment can then be assessed. An example of this type of pharmacist audit would be to identify patients taking statins who have not reached target cholesterol concentration. The findings can be discussed at a practice meeting and agreement gained on corrective action. These meetings also have an educational function.

Practice based pharmacists also have the opportunity to improve patient care through individual medication review clinics. Such clinics can cover several areas of drug treatment. They can, for example:

- identify suboptimal treatment and monitoring

- identify problems patients have with medicine taking

- provide education on disease and the treatment

- explain reasons for changes that are being made to treatment.

\section{CONCLUSION}

The pharmacy profession is moving from purely product supply to a clinical and information supply role. Proposed changes to the way the profession is funded should increase the speed of change. At present, pharmacists are not fully integrated into the primary health care team-this is in part the fault of the profession and in part the fault of primary care trusts and general practices for not making the most of the pharmacist's potential. Pharmacists' skills could be better used to help patients with their long term medicines.

\section{REFERENCE}

1 Department of Health. Pharmacy in the future-implementing the NHS plan. London: Department of Health; 2000. 